\title{
Nutritional Composition and Health Benefits of Teff (Eragrostis tef (Zucc.) Trotter)
}

\author{
Yoseph Asmelash Gebru (D), ${ }^{1}$ Desta Berhe Sbhatu $\mathbb{D}^{1},{ }^{1}$ and Kwang-Pyo Kim ${ }^{2}$ \\ ${ }^{1}$ Department of Biological and Chemical Engineering, Mekelle Institute of Technology, Mekelle University, P.O. Box 1632, \\ Mekelle, Tigray State, Ethiopia \\ ${ }^{2}$ Department of Food Science and Technology, College of Agriculture and Life Sciences, Chonbuk National University, Jeonju, \\ Jeollabuk-do 561-756, Republic of Korea \\ Correspondence should be addressed to Yoseph Asmelash Gebru; yagebru@gmail.com
}

Received 1 March 2020; Revised 14 May 2020; Accepted 21 May 2020; Published 27 September 2020

Academic Editor: Antonio Piga

Copyright (C) 2020 Yoseph Asmelash Gebru et al. This is an open access article distributed under the Creative Commons Attribution License, which permits unrestricted use, distribution, and reproduction in any medium, provided the original work is properly cited.

\begin{abstract}
Teff (Eragrostis tef (Zucc.) Trotter) (Poaceae) is an annual crop with a very tiny grain. The crop is mainly cultivated in Ethiopia and Eritrea where it is used in preparing a pancake-like staple food called injera. Teff grain is the smallest of all whole flour grains in the world with a length of about $1.0 \mathrm{~mm}$ and a width of about $0.60 \mathrm{~mm}$. The popularity of the crop is rapidly increasing throughout the world because of its attractive nutritional and functional properties. Thus, the crop is being successfully introduced and cultivated in many parts of the world including the USA, Canada, Australia, Switzerland, and the Netherlands. The growing global demand for the grain is due to its gluten-free nature, high level of essential amino acids (EAA), high mineral content, low glycemic index (GI), high crude fiber content, longer shelf life, and slow staling of its bread products compared to that of wheat, sorghum, rice, barley, and maize. The grain is linked to several health benefits including prevention and treatment of diseases such as celiac disease, diabetes, and anemia. These call for huge research opportunities to explore the nutritional and functional properties of the grain.
\end{abstract}

\section{Introduction}

Teff (Eragrostis tef (Zucc.) Trotter) (Poaceae) is an indigenous Ethiopian annual crop cultivated mainly for its small grain used in the preparation of a pancake-like staple food called injera (Amharic, Tigrinya) [1]. The genus Eragrostis consists of about 350 varieties. But teff is the only cultivated species [2]. The crop is a stress-tolerant, domesticated about 3,000 years ago. It grows in a diversity of environmental conditions extending from lowlands to highlands. Teff grain is also tolerant to storage pests [3]. Therefore, teff can be considered as a low-risk crop since it can be cultivated and thrived in a wide range of ecological conditions including under harsher environmental conditions where many other cereals fail.

Teff is more preferred by farmers and consumers over many other common Eritrean and Ethiopian grain crops such as wheat due to its agronomic traits and uses. But less is known about the crop by the academic and scientific communities outside Eritrea and Ethiopia. It is an unexplored and underexploited grain crop that needs increased attention by researchers for many purposes. Giving increased focus to such underutilized and understudied food crops like teff contributes to food and nutrition security, healthy food availability, income generation for livelihoods of small-scale farmers, and environmental services in the current depleting plant resources and increasing world population [4].

Recently, there is a growing interest on the crop since its grain is gaining global popularity for its outstanding nutritional properties [5]. It is successfully introduced and cultivated in many parts of the world including the USA, Canada, Australia, Switzerland, and the Netherlands [6]. The total population consuming teff products on daily basis in 
Ethiopia alone has currently reached more than 30 million. The most attractive property is its gluten-free nature that gives it a big potential to be utilized in a wide range of food products to help people with celiac disease [7]. Food products of teff are rich in crude fiber as the whole grain flour of the crop incorporates the bran of the grain. The grain of the crop provides relatively higher protein content with an excellent balance and a complete set of essential amino acids [8]. The grain is also high in iron content and other minerals such as calcium, copper, and zinc compared to other cereal grains consumed as whole grain flours such as wheat, maize, barley, and sorghum $[9,10]$. It was also recently proven to be a significant source of bioactive compounds including polyphenols, especially very rich in flavonoid derivatives $[6,11]$ which are rare in the other common grains.

Even though more studies are yet to be conducted, a range of health benefits have so far been established due to the grain's physical and nutritional properties. For instance, some studies reported in vitro antioxidant activities of the grain; thus, it is believed to improve the hemoglobin level in the human body and help prevent malaria, anemia, and diabetes $[5,12]$. The rich chemical composition and functional properties of the grain encouraged researchers in many laboratories across the globe to explore and develop teff food products with functional properties $[13,14]$. This review is prepared to summarize and present the latest scientific evidence regarding the nutritional compositions, functional properties, and health benefits of teff grain as well as suggest some directions for future research on its applications in the health food industries.

\section{Physical Characteristics and Nutritional Compositions of Teff Grain}

2.1. Physical Characteristics. Teff grain is the smallest of all whole flour grains in the world with a length of about $1.0 \mathrm{~mm}$ and a width of about $0.60 \mathrm{~mm}$. The average thousand grain weight of teff kernels is only $0.26 \mathrm{~g}$. The grain color ranges from a light ivory to very dark reddish brown depending on the variety (Figure 1). However, when the whole grain is finely ground, the difference in flour color becomes less noticeable. This may indicate that the pigmenting compounds of the brown teff grains are mainly accumulated on the grain pericarp.

2.2. Proteins. Cereals are alternative sources of proteins in overcoming protein deficiencies and replacing the expensive animal proteins. It is well established that the nutritional and food product qualities of cereals are mainly attributed to the physiological functions and food processing characteristics of their seed storage proteins (SSPs) [15]. Even though there are wide-ranging data on the protein and amino acid profiles of teff, some studies put the protein content of the grain from 12.8 to $20.9 \%[4,16]$. Our recent study reported a total protein content of $9.37 \%$ and an average essential amino acid content of $40.9 \%$. Of the total amino acid content, lysine constituted the highest concentration with $12.8 \mathrm{mg} / \mathrm{g}$ of flour dry weight. Regarding the SSP fractions of teff, glutelins
(46.6\%) constituted the major proteins followed by albumins (39.1\%), prolamins (29.4\%), and globulins (21.1\%) [17]. The rheological properties such as gelation, foaming, and water hydration of teff proteins were also studied in different solutions. High $\mathrm{NaCl}$ concentration of up to $1.0 \mathrm{M}$ decreased the least gelation concentration (LGC) of teff protein from 6 to $2 \%$ [18]. The foaming capacity of teff protein concentrates increased by twofold in 0.05 to $0.5 \mathrm{M} \mathrm{NaCl}$. However, there is no report that compares these properties of teff with those of other common grains. Therefore, the effects of teff proteins on the quality of food processing such as doughmaking and bread qualities in comparison with those of wheat and other common flours are interesting areas of future research.

2.3. Carbohydrates: Starch and Dietary Fiber. The tiny size of teff grain increases the surface area of its coating layer that results in a higher concentration of insoluble polysaccharides in its whole flour [19]. The total carbohydrate content of teff grain was reported to be $85.6 \%$ with starch content ranging from 74 to $75.5 \%$ [20]. Its amylose content ranged from 20 to $30 \%$ and the molecular weights of its amylopectin and amylose ranged from $10.1 \times 10^{7} \mathrm{gm} / \mathrm{mol}$ to $16.5 \times 10^{7} \mathrm{gm} / \mathrm{mol}$ and $1.0 \times 10^{6}$ to $1.3 \times 10^{6} \mathrm{gm} / \mathrm{mol}$, respectively $[21,22]$. The physicochemical studies of teff also resulted in interesting reports. The paste of teff starch had lower viscosity than that of maize starch [22]. Teff starch also showed higher water absorption index (WAI) (mean $108 \%$ ) and lower water solubility index (WSI) (mean $0.34 \%$ ) compared to maize starch. One study reported that the total dietary fiber content of whole grain teff is 9.8\% (dry basis). This is much higher than that of the common cereals such as wheat (3.4\%), rice $(0.43 \%)$, oat $(4.05 \%)$, quinoa $(7.14 \%)$, buckwheat (2.18\%), sorghum (4.51\%), and maize (2.62\%) [23]. The higher dietary fiber of teff grain is accounted to the higher proportion of bran, an important source of fiber. These imply that increased consumption of teff brings about important health benefits.

2.4. Lipids. A comparative evaluation study of lipid contents of several grains showed that teff grain flour has $4.4 \%$ lipid content. This lipid content of teff grain flour was higher than that of wheat $(3.6 \%)$, rice $(0.9 \%)$, sorghum $(3.5 \%)$, and maize $(2.5 \%)$ grain flours and lower than that of oat $(6.7 \%)$ and quinoa (8.6\%) grain flours [24]. Of the total lipid in teff, $80 \%$ of it exists in free form. This value was similarly higher than the free lipid of wheat, rye, and buckwheat. One study on teff flour using the acid hydrolysis method reported the presence of relatively lower lipid bound to starch as compared to wheat, rye, quinoa, and buckwheat flours [25]. The majority of the teff lipids were found to be unsaturated, with linoleic and oleic acids accounting for 50\% and 29\%, respectively [24]. Hence, teff can also be a good source of healthy lipids with a comparable potential as other common grains.

2.5. Bioactive Phytochemicals. Teff is rich not only in macronutrients and micronutrients but also in a variety of bioactive nonessential metabolites such as phenolic compounds and saponins. The majority of the studies in teff 


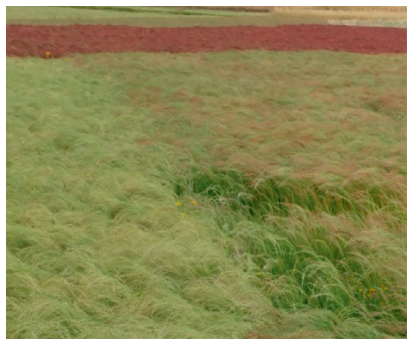

(a)

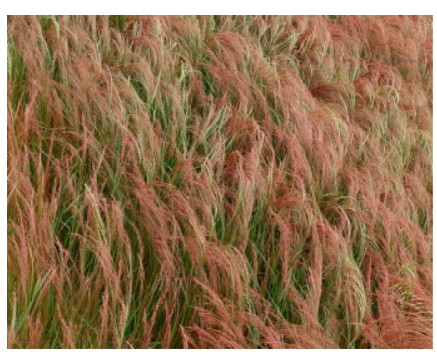

(b)

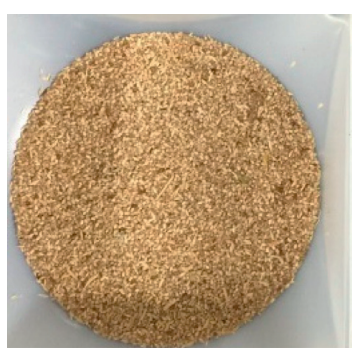

(c)

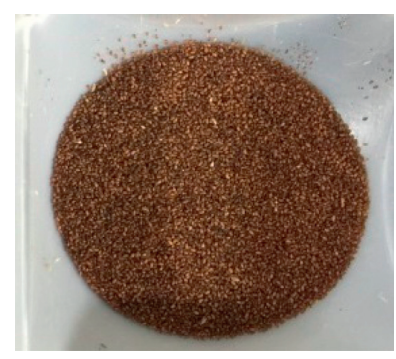

(d)

Figure 1: Teff crop field in Northern Ethiopia (Mekelle, Tigray). (a) Mature teff crop field showing its genetic diversity; (b) closer look at the grain heads; (c) white teff grains; (d) brown teff grains.

phytochemistry often focused on phenolic compounds due to their potential in reducing the risk of chronic diseases. In vitro studies on the chemical constituents of teff grain showed that the constituents have antioxidant anti-inflammatory properties and other health-promoting effects. But estimating the exact contents of the constituents such phenolic compounds is very difficult as the constituents are highly complex which can exist in free, conjugated, and bound forms $[26,27]$. One comparative analysis study on teff grain flour reported a total phenolic content (TPC) of 123.6 GAE (gallic acid equivalent)/100 g dry weight TPC. This value is much higher than the TPC of other Ethiopian staple cereals such as maize and wheat [28]. Another study analyzed the total flavonoid contents (TFCs) and TPCs of five teff varieties [29]. The TFCs and TPCs of brown teff varieties were higher than those of the white varieties. The TFCs and TPCs ranged from 0.62 to $1.16 \mathrm{mg} R E$ (rutin equivalent)/g) and 1.41 to $2.19 \mathrm{mg} \mathrm{GAE} / \mathrm{g}$, respectively. These imply that teff varieties vary widely in their phenolic contents.

Few studies attempted to identify individual phenolic compounds in teff grain. A study that identified individual molecules through mass spectroscopic tools reported that the extractable phenolic compounds in the grain were almost exclusively flavones, primarily their C-glycosides [11] (Table 1). The results show that there is a distinct difference in phenolic profile between white and brown teff varieties, where the white varieties contain only apigenin glycosides and the brown varieties mostly contain luteolin glycosides. The higher concentrations of flavones in teff compared to other common cereals become the ground to suggest that the grain can be applied as a valuable source of rare dietary phenolic compounds.

2.6. Minerals. A comparative mineral content analysis of teff grain showed that $\mathrm{P}, \mathrm{Mg}, \mathrm{Mn}$, and $\mathrm{Cu}$ are present in higher concentrations in the grain than in other common cereals like maize and wheat [28]. Data from the Agricultural Research Service Database of the United States Department of Agriculture showed that the mineral contents of the grain on a wet basis are $\mathrm{Ca}(180 \mathrm{mg} / 100 \mathrm{~g}), \mathrm{Fe}(7.63 \mathrm{mg} / 100 \mathrm{~g}), \mathrm{Mg}$ $(184 \mathrm{mg} / 100 \mathrm{~g}), \mathrm{P}(429 \mathrm{mg} / 100 \mathrm{~g}), \mathrm{K}(427 \mathrm{mg} / 100 \mathrm{~g}), \mathrm{Na}$ $(12 \mathrm{mg} / 100 \mathrm{~g})$, and $\mathrm{Zn}(3.63 \mathrm{mg} / 100 \mathrm{~g})$ [30]. Variations in mineral contents have been reported between brown and white teff grain varieties. The brown teff varieties were found to have a higher $\mathrm{Fe}$ and $\mathrm{Ca}$ content than the white varieties. On the other hand, the white teff varieties have higher $\mathrm{Cu}$ content than the brown varieties [31]. However, the bioavailability of most minerals is affected by chelating components such as phytic acid. This can be a nutritional concern in the grain as its whole grain phytic acid content is estimated to be up to $1,544 \mathrm{mg} / 100 \mathrm{~g}$ dry weight [32]. Hence, processing methods such as fermentation of teff batter with lactic acid bacteria and fungi are believed to enhance the bioavailability of teff minerals.

2.7. Vitamins. The vitamin composition and contents in teff are yet to be experimentally explored. The USDA Food Composition Databases [30] included the contents of some vitamins. Raw teff has niacin $(3.363 \mathrm{mg} / 100 \mathrm{~g})$, vitamin $\mathrm{B} 6$ $(0.482 \mathrm{mg} / 100 \mathrm{~g})$, thiamin $(0.39 \mathrm{mg} / 100 \mathrm{~g})$, riboflavin $(0.27 \mathrm{mg} / 100 \mathrm{~g})$, vitamin $\mathrm{K}$ (phylloquinone) $(1.9 \mu \mathrm{g} / 100 \mathrm{~g})$, vitamin A (9 IU), and $\alpha$-tocopherol $(0.08 \mathrm{mg} / 100 \mathrm{~g})$.

\section{Significance of Teff on Public Health}

3.1. Celiac Disease. Celiac disease is a disease of the digestive system characterized by abnormal nutrient adsorption in the inner lining of the small intestine due to intolerance to gluten, a protein found in wheat, barley, and rye [33]. The disease affects about $1 \%$ of the world population [31]. The prevalence of celiac disease increases with conditions such as type 1 diabetics and symptomatic iron deficiency anemia. The disease affects 3 to $6 \%$ of people with type 1 diabetics and 10 to $15 \%$ of people with symptomatic iron deficiency anemia [34]. The only effective and safe treatment available for those affected by celiac disease so far is a strict and lifelong gluten-free diet [35]. The fact that the most common and abundant food products are made from gluten-containing cereals like wheat makes following a strict gluten-free diet difficult. Hence, formulating gluten-free food products is an interesting subject for food technologists and nutritionists. But many of the gluten-free products such as quinoa and amaranth cannot meet the recommended daily nutrient intake of celiac disease patients. The energy intake of such patients may also be limited to only animal fats. Therefore, these limitations are solved by teff-based gluten-free food products. Teff, as nutrient-rich gluten-free grain, is being frequently used as the best choice for celiac disease patients 
TABLE 1: Concentration of tentatively identified flavones in white and brown teff grains.

\begin{tabular}{|c|c|c|c|}
\hline \multirow{2}{*}{ Variety } & \multirow{2}{*}{ Tentatively identified compounds } & \multicolumn{2}{|c|}{ Concentration ( $\mu \mathrm{g} / \mathrm{g}$ dry weight) } \\
\hline & & Sample 1 & Sample 2 \\
\hline \multirow{5}{*}{ White } & Apigenin-6,8-C-diglucoside & $130.8 \pm 08.9$ & $121.9 \pm 10.9$ \\
\hline & Apigenin-8-C-glucosyl-7-O-glucoside & $209.4 \pm 12.0$ & $113.6 \pm 04.9$ \\
\hline & Apigenin-6-C-glucosyl-2"-O-glucoside & $590.4 \pm 13.9$ & $375.1 \pm 05.7$ \\
\hline & Apigenin-8-C-glucoside & $388.3 \pm 07.4$ & $195.2 \pm 03.9$ \\
\hline & Apigenin-7-O-(6"-syringyl) glucosyl-8-C-arabinoside & $252.4 \pm 06.2$ & $168.7 \pm 03.6$ \\
\hline \multirow{5}{*}{ Brown } & Luteolin-8-C-glucosyl-7-O-glucoside & $148.6 \pm 08.3$ & $190.5 \pm 01.1$ \\
\hline & Luteolin-6-C-glucosyl-7-O-glucoside isomer & $599.3 \pm 32.9$ & $570.9 \pm 10.0$ \\
\hline & Luteolin-8-C-glucosyl-7-O-neohesperidoside & $107.9 \pm 09.8$ & $93.3 \pm 01.5$ \\
\hline & Luteolin-8-C-glucoside & $204.8 \pm 14.4$ & $250.5 \pm 19.4$ \\
\hline & Luteolin-7-O-(2"-syringyl) arabinosyl-6-C-glucoside & $288.2 \pm 23.5$ & $332.6 \pm 21.0$ \\
\hline
\end{tabular}

Ravisankar et al. [11].

without fortification in countries like the Netherlands [35]. Moreover, teff grain is a versatile one with a preferable taste and texture that can be used in making a variety of food products including Sourdough bread, non-Sourdough bread, cookies, pasta, weaning food, and extruded product as well as beverages like lactic acid beverages and beer $[5,36-38]$.

3.2. Diabetes. Diabetes mellitus, commonly known as diabetes, is a metabolic disorder characterized by increased blood glucose level and abnormal insulin regulation. New trends of the modern world towards high-fat diet, prevalence of obesity, and increased sedentary lifestyle are leading to a global increase in diabetes incidence [39]. It has become a major global health problem with a whopping 415 million diabetic patients in the world according to estimates in 2015 [40]. The quantity and quality of carbohydrates in diet are the primary determinants of glucose levels and insulin responses in the human body [41].

Glycemic index (GI) reflects the postprandial glucose response of carbohydrate-containing foods and its effect is considered to be closely related to the fiber content of the foods consumed $[42,43]$. One recent study reported that low GI foods can reduce the risk of type 2 diabetes [44]. These imply that the type of carbohydrate in the food plays a central role in the onset and prevention of diabetes. Teff has relatively higher crude fiber content compared to other common grains and lower GI [45]. Therefore, teff-based foods are expected to have outstanding contributions to the prevention and amelioration of diabetes. Moreover, the high concentration of calcium in the grain can prevent the onset of disorders associated with low calcium consumption like weight gain, accumulation of fat, and osteoporosis that often lead to diabetes. The absence of empirical evidence on the effects of teff-based diet on diabetes calls for future research.

3.3. Anemia. Anemia is a condition where the amount of red blood cells, which reflects the hemoglobin content, decreases leading to impaired capacity of the blood to transport oxygen in the body. Anemia affects more than $25 \%$ of the world population with higher frequencies in the developing countries [46]. The deficiency of dietary iron is the principal cause of anemia throughout the world. Iron deficiency anemia has particularly a substantial effect on the lives of young children and premenopausal women in both lowincome and developed countries [47]. While food supplements and fortifications increase the dietary intake of iron, the strategies often have gastrointestinal tract-specific side effects including constipation, nausea, and diarrhea [46]. Hence, dietary iron intake through the consumption of naturally iron-rich plants such as teff can be considered as a more efficient and safer strategy. In fact, a twofold increase in iron content was observed in wheat bread prepared with $30 \%$ teff flour. This indicates that wheat bread diet consisting of $30 \%$ of teff is able to maintain the standard serum iron levels in pregnant women [46]. With these promising observations, future research may focus on the beneficial effects of teff consumption on the cardiovascular disorders, most importantly on heart failure which is often linked to iron deficiency.

\section{Prospects for Further Exploration}

The growing interest in the food and beverage products designated as health foods in the market embodies a new development of consumer behavior. The interest of the present health-conscious society is leading to the growing demand for foods with additional functional properties. This, in turn, is driving the academic and research communities towards exploring diet formulations with potential health benefits. The lack of detailed account on the functional properties of teff in the literature prevents us from establishing a comprehensive understanding of the mechanisms by which it promotes health. Hence, further studies are required on the specific functional properties of the grain in order to be fully exploited in the health food industries. Since teff grain is mostly used as fermented foods, its functional properties can be enhanced through bacteria and yeast-mediated fermentations. This is an interesting area of future research. Establishing standards for the quality and quantity of starter culture, fermentation conditions, and other ingredients can generate more data on its biological activities and potential applications.

The popularity of teff is growing steadily in North America, Europe, and Australia. Since the crop is well- 
adapted to a diversity of ecological and environmental conditions, studies in its agronomic features can help in expanding it to new places all over the world as a sustainable crop and a popular food. The relatively lower GI of teff hints its potential therapeutic effects on diabetes. Studies on the effects of teff grains can result in data that help in the formulation of food products with health benefits for patients with diabetics as well as other hyperglycemic and hypoglycemic diseases.

\section{Data Availability}

All data generated or analyzed during this study are included in this article.

\section{Conflicts of Interest}

The authors declare that they have no conflicts of interest.

\section{References}

[1] E. K. Arendt and E. Zannini, "Teff. cereal grains for the food and beverage industries," in Woodhead Publishing Series in Science, Technology and Nutrition, pp. 351-369e, Elsevier, Amsterdam, The Netherlands, 2013.

[2] K. Habtegebrial, B. R. Singh, and M. Haile, "Impact of tillage and nitrogen fertilization on yield, nitrogen use efficiency of tef (Eragrostis tef (Zucc.) Trotter) and soil properties," Soil and Tillage Research, vol. 94, no. 1, pp. 55-63, 2007.

[3] D. Girma, K. Assefa, S. Chanyalew, G. Cannarozzi, C. Kuhlemeier, and Z. Tadele, "The origins and progress of genomics research on tef (Eragrostis tef)," Plant Biotechnology Journal, vol. 12, no. 5, pp. 534-540, 2014.

[4] F. Zhu, "Chemical composition and food uses of teff (Eragrostis tef)," Food Chemistry, vol. 239, pp. 402-415, 2018.

[5] M. M. Gebremariam, M. Zarnkow, and T. Becker, "Teff (Eragrostis tef) as a raw material for malting, brewing and manufacturing of gluten-free foods and beverages: a review," Journal of Food Science and Technology, vol. 51, no. 11, pp. 2881-2895, 2014.

[6] H. Shumoy and K. Raes, "Antioxidant potentials and phenolic composition of tef varieties: an indigenous ethiopian cereal," Cereal Chemistry Journal, vol. 93, no. 5, pp. 465-470, 2016.

[7] K. O. Nascimento, S. N. D. Paes, I. Reis de Oliveira, I. P. Reis, and I. M. Augusta, "Teff: suitability for different food applications and as a raw material of gluten-free: a literature review," Journal of Food and Nutrition Research, vol. 6, no. 2, pp. 74-81, 2018.

[8] W. Abebe, C. Collar, and F. Ronda, "Impact of variety type and particle size distribution on starch enzymatic hydrolysis and functional properties of tef flours," Carbohydrate Polymers, vol. 115, pp. 260-268, 2015.

[9] E. Campo, L. del Arco, L. Urtasun, R. Oria, and A. FerrerMairal, "Impact of Sourdough on sensory properties and consumers' preference of gluten-free breads enriched with teff flour," Journal of Cereal Science, vol. 67, pp. 75-82, 2016.

[10] Y. Abebe, A. Bogale, K. M. Hambidge, B. J. Stoecker, K. Bailey, and R. S. Gibson, "Phytate, zinc, iron and calcium content of selected raw and prepared foods consumed in rural Sidama, Southern Ethiopia and implications for bioavailability," Journal of Food Composition and Analysis, vol. 20, no. 3-4, pp. 161-168, 2007.
[11] S. Ravisankar, K. Abegaz, and J. M. Awika, "Structural profile of soluble and bound phenolic compounds in teff (Eragrostis tef) reveals abundance of distinctly different flavones in white and brown varieties," Food Chemistry, vol. 263, pp. 265-274, 2018.

[12] S. O. Salawu, M. J. Bester, and K. G. Duodu, "Phenolic composition and bioactive properties of cell wall preparations and whole grains of selected cereals and legumes," Journal of Food Biochemistry, vol. 38, no. 1, pp. 62-72, 2014.

[13] G. Giuberti, A. Gallo, L. Fiorentini, P. Fortunati, and F. Masoero, "In vitro starch digestibility and quality attributes of gluten free "tagliatelle" prepared with teff flour and increasing levels of a new developed bean cultivar," Starch-Stärke, vol. 68, no. 3-4, pp. 374-378, 2016.

[14] M. M. Gebremariam, A. Hassani, M. Zarnkow, and T. Becker, "Investigation of fermentation conditions for teff (Eragrostis tef) malt-wort by Lactobacillus amylolyticus," LWT-Food Science and Technology, vol. 61, no. 1, pp. 164-171, 2015.

[15] S. Gorinstein, E. Pawelzik, E. Delgado-Licon, R. Haruenkit, M. Weisz, and S. Trakhtenberg, "Characterisation of pseudocereal and cereal proteins by protein and amino acid analyses," Journal of the Science of Food and Agriculture, vol. 82, no. 8, pp. 886-891, 2002.

[16] T. S. El-Alfy, S. M. Ezzat, and A. A. Sleem, "Chemical and biological study of the seeds of Eragrostis tef (Zucc.) Trotter," Natural Product Research, vol. 26, no. 7, pp. 619-629, 2012.

[17] Y. A. Gebru, J. Hyun-II, K. Young-Soo, K. Myung-Kon, and K. Kwang-Pyo, "Variations in amino acid and protein profiles in white versus brown teff (Eragrostis tef) seeds, and effect of extraction methods on protein yields," Foods, vol. 8, no. 6, p. 202, 2019.

[18] L. A. Arogundade, "Functional characterization of tef (Eragostics tef) protein concentrate: influence of altered chemical environment on its gelation, foaming, and water hydration properties," Food Hydrocolloids, vol. 20, no. 6, pp. 831-838, 2006.

[19] A. Marti, M. Marengo, F. Bonomi et al., "Molecular features of fermented teff flour relate to its suitability for the production of enriched gluten-free bread," $L W T$, vol. 78, pp. 296-302, 2017.

[20] W. Abebe and F. Ronda, "Rheological and textural properties of tef [Eragrostis tef (Zucc.) Trotter] grain flour gels," Journal of Cereal Science, vol. 60, no. 1, pp. 122-130, 2014.

[21] G. Bultosa, B. R. Hamaker, and J. N. Bemiller, "An SEC-MALLS study of molecular features of water-soluble amylopectin and amylose of tef [Eragrostis tef (Zucc.) Trotter] starches," Starch-Stärke, vol. 60, no. 1, pp. 8-22, 2008.

[22] G. Bultosa, A. N. Hall, and J. R. N. Taylor, "Physico-chemical characterization of grain tef [Eragrostis tef (Zucc.) Trotter] starch," Starch-Stärke, vol. 54, no. 10, pp. 461-468, 2002.

[23] A.-S. Hager, A. Wolter, F. Jacob, E. Zannini, and E. K. Arendt, "Nutritional properties and ultra-structure of commercial gluten free flours from different botanical sources compared to wheat flours," Journal of Cereal Science, vol. 56, no. 2, pp. 239-247, 2012.

[24] A.-S. Hager, M. Czerny, J. Bez, E. Zannini, and E. K. Arendt, "Starch properties, in vitro digestibility and sensory evaluation of fresh egg pasta produced from oat, teff and wheat flour," Journal of Cereal Science, vol. 58, no. 1, pp. 156-163, 2013.

[25] C. Collar and A. Angioloni, "Pseudocereals and teff in complex breadmaking matrices: impact on lipid dynamics," Journal of Cereal Science, vol. 59, no. 2, pp. 145-154, 2014. 
[26] A. Valdés, L. Vidal, A. Beltrán, A. Canals, and M. C. Garrigós, "Microwave-assisted extraction of phenolic compounds from almond skin byproducts (Prunus amygdalus): a multivariate analysis approach," Journal of Agricultural and Food Chemistry, vol. 63, no. 22, pp. 5395-5402, 2015.

[27] A. M. Gómez-Caravaca, A. Segura-Carretero, A. FernándezGutiérrez, and M. F. Caboni, "Simultaneous determination of phenolic compounds and saponins in quinoa (Chenopodium quinoaWilld) by a liquid chromatography-diode array detection-electrospray ionization-Time-of-flight mass spectrometry methodology," Journal of Agricultural and Food Chemistry, vol. 59, no. 20, pp. 10815-10825, 2011.

[28] S. F. Forsido, H. P. V. Rupasinghe, and T. Astatkie, "Antioxidant capacity, total phenolics and nutritional content in selected ethiopian staple food ingredients," International Journal of Food Sciences and Nutrition, vol. 64, no. 8, pp. 915-920, 2013.

[29] E. Kotásková, D. Sumczynski, J. Mlček, and P. Valášek, "Determination of free and bound phenolics using HPLC$\mathrm{DAD}$, antioxidant activity and in vitro digestibility of Eragrostis tef," Journal of Food Composition and Analysis, vol. 46, pp. 15-21, 2016.

[30] USDA Food Composition Databases, FoodData Central, USDA, Washington, DC, USA, 2017.

[31] N. Gujral, H. J. Freeman, and A. B. R. Thomson, "Celiac disease: prevalence, diagnosis, pathogenesis and treatment," World Journal of Gastroenterology, vol. 18, no. 42, pp. 60366059, 2012.

[32] K. Baye, C. Mouquet-Rivier, C. Icard-Vernière, C. Picq, and J.-P. Guyot, "Changes in mineral absorption inhibitors consequent to fermentation of Ethiopianinjera: implications for predicted iron bioavailability and bioaccessibility," International Journal of Food Science \& Technology, vol. 49, no. 1, pp. 174-180, 2014.

[33] P. H. R. Green and C. Cellier, "Celiac disease," New England Journal of Medicine, vol. 357, no. 17, pp. 1731-1743, 2007.

[34] A. Pham-Short, K. C. Donaghue, G. Ambler, H. Phelan, S. Twigg, and M. E. Craig, "Screening for celiac disease in type 1 diabetes: a systematic review," Pediatrics, vol. 136, no. 1 , pp. e170-e176, 2015.

[35] K. A. Bascuñán, M. C. Vespa, and M. Araya, "Celiac disease: understanding the gluten-free diet," European Journal of Nutrition, vol. 56, no. 2, pp. 449-459, 2017.

[36] C. M. Mancebo, J. Picón, and M. Gómez, "Effect of flour properties on the quality characteristics of gluten free sugarsnap cookies," LWT-Food Science and Technology, vol. 64, no. 1, pp. 264-269, 2015.

[37] F. Ronda, W. Abebe, S. Pérez-Quirce, and C. Collar, "Suitability of tef varieties in mixed wheat flour bread matrices: a physico-chemical and nutritional approach," Journal of Cereal Science, vol. 64, pp. 139-146, 2015.

[38] A.-S. Hager, F. Lauck, E. Zannini, and E. K. Arendt, "Development of gluten-free fresh egg pasta based on oat and teff flour," European Food Research and Technology, vol. 235, no. 5, pp. 861-871, 2012.

[39] L. Guariguata, D. R. Whiting, I. Hambleton, J. Beagley, U. Linnenkamp, and J. E. Shaw, "Global estimates of diabetes prevalence for 2013 and projections for 2035," Diabetes Research and Clinical Practice, vol. 103, no. 2, pp. 137-149, 2014.

[40] K. Ogurtsova, J. D. da Rocha Fernandes, Y. Huang et al., "IDF Diabetes Atlas: global estimates for the prevalence of diabetes for 2015 and 2040," Diabetes Research and Clinical Practice, vol. 128, pp. 40-50, 2017.
[41] T. M. S. Wolever, "Dietary carbohydrates and insulin action in humans," British Journal of Nutrition, vol. 83, no. S1, pp. S97-S102, 2000.

[42] M. D. Gutschall, C. K. Miller, D. C. Mitchell, and F. R. Lawrence, "A randomized behavioural trial targeting glycaemic index improves dietary, weight and metabolic outcomes in patients with type 2 diabetes," Public Health Nutrition, vol. 12, no. 10, pp. 1846-1854, 2009.

[43] T. M. Wolever, "Relationship between dietary fiber content and composition in foods and the glycemic index," The American Journal of Clinical Nutrition, vol. 51, no. 1, pp. 72-75, 1990.

[44] L. S. A. Augustin, C. W. C. Kendall, D. J. A. Jenkins et al., "Glycemic index, glycemic load and glycemic response: an international scientific consensus summit from the international carbohydrate quality consortium (ICQC)," Nutrition, Metabolism and Cardiovascular Diseases, vol. 25, no. 9, pp. 795-815, 2015.

[45] A. Wolter, A.-S. Hager, E. Zannini, and E. K. Arendt, "In vitro starch digestibility and predicted glycaemic indexes of buckwheat, oat, quinoa, sorghum, teff and commercial glutenfree bread," Journal of Cereal Science, vol. 58, no. 3, pp. 431-436, 2013.

[46] N. J. Kassebaum, R. Jasrasaria, M. Naghavi et al., "A systematic analysis of global anemia burden from 1990 to 2010," Blood, vol. 123, no. 5, pp. 615-624, 2014.

[47] E. McLean, M. Cogswell, I. Egli, D. Wojdyla, and B. De Benoist, "Worldwide prevalence of anaemia, WHO vitamin and mineral nutrition information system, 1993-2005," Public Health Nutrition, vol. 12, no. 4, pp. 444-454, 2009. 